www.jmscr.igmpublication.org Impact Factor 5.244

Index Copernicus Value: 83.27 ISSN (e)-2347-176x ISSN (p) 2455-0450 crossref DOI: _https://dx.doi.org/10.18535/jmscr/v4i11.53

\title{
Anti Mullerian Hormone Levels and Antral Follicle Count as Predictors of
}

\section{In Vitro Fertilisation Outcomes}

\author{
Authors

\section{Dr Basavanapalli Menaka ${ }^{1}$, Dr Vasundhara Kamineni ${ }^{2}$,}

\section{Dr Akkenapally Prasanna Latha ${ }^{3}$, Dr Nandini Joshi ${ }^{4}$, Dr Vamsha Shree ${ }^{5}$}

${ }^{1}$ Assistant Professor, Dept of Obstetrics and Gynecology, Kamineni Academy of Medical Sciences and Research Centre, LB Nagar, Hyderabad, Telangana, India

Email: menakameghareddy@gmail.com

${ }^{2}$ Professor and HOD, Dept of Obstetrics and Gynecology, Kamineni Academy of Medical Sciences and

Research Centre, LB nagar, Hyderabad, Telangana, India

Email:vparliker@yahoo.com

${ }^{3}$ Assistant professor, Dept of Obstetrics and Gynecology, Kamineni Academy of medical sciences and

Research Centre, LB nagar, Hyderabad, Telangana, India

Email:drlatasri@gmail.com

${ }^{4}$ Assistant professor, Dept of Obstetrics and Gynecology, Kamineni Academy of medical sciences and

Research Centre, LB nagar, Hyderabad, Telangana, India

Email: jahagirdar.nandini@gmail.com

${ }^{5}$ Junior Resident, Dept of Obstetrics and Gynecology, Kamineni Academy of medical sciences and Research

Centre, LB nagar, Hyderabad, Telangana, India

Email: jahagirdar123@yahoo.co.in

Corresponding Author

\section{Dr Basavanapalli Menaka}

11-14-262/C5/201-1, Chitra Layout, LB Nagar, Hyderabad, 500074

\section{ABSTRACT}

Objective: To study the role of Anti Mullerian hormone levels and antral follicle count in predicting the outcomes in IVF cycles.

Methods: A prospective study was done in fifty women with infertility undergoing IVF treatment. On Day3, serum levels of E2, FSH, AMH were measured and TVS for AFC was done. Ovarian stimulation was started using HMG from day2 of menstrual cycle. Follicular growth monitored. All patients were categorized into 4 fertility groups based on their AMH and AFC values as optimal, satisfactory, low and very low fertility potential groups. IVF/ICSI outcomes were measured in each category and tabulated.

Results: In our study 29(58\%) pregnancies were obtained out of 50 women. There was no significant difference 
in terms of mean age, duration of infertility and BMI b/w pregnant and non pregnant women ( $p$ value0.194,0.367,0.475). Statistical significance was seen in AMH levels, AFC, between pregnant and non pregnant women ( $p$ value- $0.003,0.0001$ ). There is positive correlation between AMH levels \& AFC, serum AMH, AFC and pregnancy status. Mean serum AMH in pregnant group is 5.1024, and in non pregnant group it is 2.2738. Mean AFC in pregnant group is double that of non pregnant group i.e. 7.31 and 3.48, which suggests that both are good predictors of pregnancy outcome in IVF cycles.

Conclusions: Serum AMH levels and AFC are good predictors of pregnancy outcomes in IVF/ICSI cycles. Clinical pregnancy outcome is maximum in low and satisfactory fertility potential groups i.e .,with serum AMH 2.2 to 6.7 and $A F C$ between 3 to 10.

\section{INTRODUCTION}

Infertility affects approximately $13-14 \%$ of couples in reproductive age group. It is defined as the inability to conceive after 1 year of properly timed, unprotected intercourse. This definition is based on the cumulative probability of pregnancy ${ }^{1}$. Assisted reproductive technologies (ART) have enabled millions of people in the world to have biological children who otherwise would not have been able to do so. According to the European Society for Human Reproduction and Embryology, more than three million babies have been born using ART worldwide in the last 30 years, enabling infertile women and men; single women and men; to form genetically-related families.

The reproductive capacity of a woman depends on many factors. Prediction of ovarian reserve has long been the golden key of reproductive endocrinology. Various endocrine [follicle stimulating hormone (FSH), inhibin B, estradiol

(E2) etc.], and ultrasound tests [ovarian volume, antral follicle counts (AFC)] have been suggested to improve prediction of oocyte yield and pregnancy outcome following assisted reproductive technologies (ART) . Currently, most in vitro fertilization (IVF) clinicians determine starting doses of gonadotropin in the first cycle of IVF based principally on the patient's age and basal FSH levels.

The success of assisted reproductive technology programme depends on adequate ovarian reserve. $^{2}$
Though ovarian reserve declines with age, it varies between individuals. ${ }^{3,4}$

The established predictors of reproductive potential during infertility treatment are maternal age, early follicular phase FSH concentrations, and less popularly, serum inhibin B concentration. None of these particularly are reliable predictors of the number or quality of oocytes remaining within the ovary, or the capsule. The pregnancy rate tends to increase as AMH increases, although this remains non-significant. Recently, interest in the use of anti-müllerian hormone (AMH) and AFC to predict patient response to ovarian stimulation has been intense. ${ }^{5,6}$

A relatively new marker, $\mathrm{AMH}$, was first identified as a specific protein in Sertoli cells of fetal testis, which inhibits the development of the mullerian duct .AMH, a member of the transforming growth factor-beta super-family, is only produced by the granulosa cells surrounding preantral and small antral follicles in the ovary . AMH has been shown to decrease the sensitivity of preantral and small antral follicles to FSH, and its production is independent from that of FSH and reflects only the follicle population. ${ }^{7} \mathrm{AMH}$ expression decreases during the FSH-dependent final stages of follicular growth and atretic follicles do not express AMH. As the woman approaches menopause there is linear decline of AMH levels over time. ${ }^{8,9}$ Body mass index (BMI) does not seem to have an effect on serum AMH levels in reproductive age women, both with and 
without polycystic ovary syndrome (PCOS). There have been several studies about the relationship between AMH levels and oocyte or embryo quality. Feyerisen et al, demonstrated that AMH had a strongest relationship with AFC than other typical biomarkers. ${ }^{10}$ The aim of this study is to investigate the role of $\mathrm{AMH}$ levels and AFC in predicting the outcomes in IVF.

\section{MATERIALS AND METHODS}

This was a prospective Observational Study conducted from october 2013 to september 2014 in the department of obstetrics and gynaecology, Kamineni hospital, hyderabad. A total of 50 infertile women undergoing IVF treatment were included. Women with ovarian abnormalities (inability to visualise ovaries on TVS, previous oophorectomy), premature ovarian failure and couples with male factor infertility (Surgical sperm retrieval for azoospermia) were excluded from the study.

Ethical clearance was obtained from the institutional ethics committee. Informed consent as per the standard protocol was taken from all patients undergoing IVF. After history, detailed clinical examination was done and all women were subjected to routine investigations and hormonal evaluation which included serum levels of E2, FSH, AMH on day 3 of menstrual cycle. Transvaginal scan was done on day 3 of menstrual cycle to look for Antral Follicle Count .Semen analysis of the partners of all these women were within normal limits.Ovarian Stimulation was started using Human Menopausal Gonadotropin from day 2 of menstrual cycle.

Follicular growth monitoring was carried out by TVS beginning on day 7 of HMG stimulation. When more than 3 follicles larger than $18 \mathrm{~mm}$ diameter detected, 10,000IU HCG was administered intra muscularly.36hours later follicles were retrieved under general anaesthesia by TVS guided aspiration. Mature oocytes were retrieved from follicular fluid, and fertilized immediately by mature sperms using IVF after fertilization. Embryos were evaluated microscopically and best quality embryos selected for transfer.

IVF outcomes analysed for number of oocytes aspirated after controlled ovarian stimulation and number of oocytes fertilized by IVF and number of pregnancies. All patients were categorized into 4 fertility potential groups based on their AMH levels and AFC (1) Optimal fertility: AMH $>6.8 \mathrm{ng} / \mathrm{ml}$ and AFC .12. (2) Satisfactory fertility: AMH 4.0-6.7ng/ml and AFC 6-10. (3) Low fertility: AMH 2.2-3.9ng/ml and AFC 3-5. (4) Very low fertility: AMH 0.0-2.1ng/ml and AFC 02.Patients were followed up with UPT after 16days of embryo transfer and pregnant women were offered an ultrasonography 10-14days later to confirm intrauterine pregnancy and number of gestation sacs present.

The data has been presented as the arithmetical means and the standard deviations are calculated for each group as well. An independent sample ttest was performed for evaluating the statistical relations between the sub groups. A p-value $<0.05$ was considered statistically significant. All statistical analyses were performed using the statistical package for the social sciences(SPSS) soft ware version.

\section{RESULTS}

Out of 50 selected infertile women-, 8 belong to optimal fertility potential (16\%), 10 belong to satisfactory fertility potential (20\%), 15 belong to low fertility potential (30\%) and 17 belong to very low fertility potential group (34\%).

The mean age (years) of the subjects studied was 31.41. The mean AMH levels were $5.10 \mathrm{ng} / \mathrm{mL}$ in 


\section{JMSCR Vol||04||Issue||11||Page 13820-13826||November}

women who conceived and $2.27 \mathrm{ng} / \mathrm{mL}$ in women who did not (Table 1). There is significant difference between pregnant and non pregnant women serum AMH levels since p value is 0.003 . Out of 50 study subjects, 29 women became pregnant $(58 \%)$. Out of 29 pregnant women, one was < 24years age (3.4\%), 24 women were in 25 to 36years age group $(82.8 \%)$ and 4 women were >36years age (13.8\%). The mean BMI of pregnant and non pregnant women was $26.53 \mathrm{~kg} / \mathrm{m}^{2}$ and $26.81 \mathrm{~kg} / \mathrm{m}^{2}$ respectively. Among the pregnant women, 2 were with very low fertility potential $(6.9 \%), 11$ were in low potential $(37.9 \%), 10$ were in satisfactory potential $(34.5 \%)$ and 6 were in optimal potential group (20.7\%) (Table 2).The mean Antral follicle count was 7.31 and 3.48 in women who were pregnant and nonpregnant respectively (Table 3). There is significant difference in AFC between pregnant and non pregnant, since $\mathrm{p}$-value is 0.0001 .

There is significant difference in the number of oocytes retieved between pregnant and nonpregnant women(Table 4), since p-value is 0.002 is less than 0.05 ( $5 \%$ level of significance).There is positive correlation between serum $\mathrm{AMH}$ and AFC and correlation of Sr.AMH and pregnancy .

TABLE 1 Serum AMH levels and pregnancy status

\begin{tabular}{|ll|c|c|c|c|}
\hline & Pregnancy status & $\mathrm{N}$ & Mean & Std. Deviation & Std. Error Mean \\
\hline \multirow{2}{*}{ Sr.AMH } & No & 21 & 2.2738 & 2.51444 & .54870 \\
& Yes & 29 & 5.1024 & 3.51201 & .65216 \\
\hline
\end{tabular}

TABLE 2 Serum AMH Cat and pregnancy status

\begin{tabular}{|c|c|c|c|c|}
\hline & \multicolumn{2}{|c|}{ Pregnancy status } & \multirow[t]{2}{*}{ Total } \\
\hline & & Yes & No & \\
\hline \multirow[t]{4}{*}{ Sr.AMH Cat } & Very low & $2(6.9 \%)$ & $16(76.2 \%)$ & $18(36 \%)$ \\
\hline & Low & $11(37.9 \%)$ & $3(14.3 \%)$ & $14(28 \%)$ \\
\hline & Satisfactory & $10(34.5 \%)$ & $1(4.8 \%)$ & $11(22 \%)$ \\
\hline & Optimal & $6(20.7 \%)$ & $1(4.8 \%)$ & $7(14 \%)$ \\
\hline Total & & $29(100 \%)$ & $21(100 \%)$ & $50(100 \%)$ \\
\hline
\end{tabular}

TABLE 3 AFC and pregnancy status

\begin{tabular}{|ll|c|c|c|c|}
\hline & Pregnancy status & N & Mean & Std. Deviation & Std. Error Mean \\
\hline AFC & No & 21 & 3.48 & 3.311 & .722 \\
& Yes & 29 & 7.31 & 3.424 & .636 \\
\hline
\end{tabular}

TABLE 4 Pregnancy status * No. of Oocytes Retrieved

\begin{tabular}{|c|c|c|c|c|c|c|c|c|}
\hline \multirow[t]{2}{*}{ Count } & & \multicolumn{6}{|c|}{ No. of Oocytes Retrieved } & \multirow[b]{2}{*}{ Total } \\
\hline & & 1 & 2 & 3 & 4 & 5 & 6 & \\
\hline \multirow[t]{2}{*}{ Pregnancy status } & No & 2 & 13 & 0 & 3 & 3 & 0 & 21 \\
\hline & Yes & 0 & 6 & 9 & 3 & 5 & 6 & 29 \\
\hline Total & & 2 & 19 & 9 & 6 & 8 & 6 & 50 \\
\hline
\end{tabular}




\section{DISCUSSION}

The value of AMH levels and AFC in the prediction of pregnancy has been investigated in various studies which showed inconsistent results. Few studies suggest that serum AMH level is associated with pregnancy rates; whereas others suggested that serum AMH levels are not associated with pregnancy outcomes. Other markers such as AFC and inhibin B, which were thought to predict pregnancy, were also evaluated in many studies. In our study we detected that day 3 serum AMH, AFC measurements were found to be associated with pregnancy rates. Hazout ${ }^{11}$ et al. evaluated 109 women (<42 years old) and demonstrated that day 3 serum AMH level and IVF outcome were strongly associated, and higher AMH concentrations were associated with a higher clinical pregnancy rate; moreover, they showed that AMH might offer greater prognostic value than other currently available serum markers of ART outcome. In our study serum AMH was highest in satisfactory (34.5\%) and low fertility potential $(37.9 \%)$ groups among the pregnant. Eldar-Geva ${ }^{12}$ et al. concluded that serum follicular or luteal phase AMH is the only predictor for the pregnancy that had a prospective design with 56 women. $\mathrm{Wu}^{13}$ et al. detected that day $3 \mathrm{AMH}$ and AFC were significantly higher in pregnant women compared to non-pregnant women (total of 60 infertile women). Multiple regression analysis for prediction of pregnancy showed day $3 \mathrm{AMH}$ to be a good predictor of clinical pregnancy. In our study AMH and AFC levels were higher among the satisfactory and low fertility potential groups and there is significant correlation between serum AMH, AFC and pregnancy outcomes. Majumder ${ }^{1}$ et al. prospectively evaluated 162 infertile women and observed that both day $3 \mathrm{AMH}$ and AFC had highly significant correlations with the number of oocytes retrieved and the number of oocytes fertilized. AMH was better than AFC in terms of predicting live birth, but both markers were more valuable in predicting the absence rather than the occurrence of live birth. In our study we evaluated 50 infertile women and observed that serum AMH and AFC had significant correlations with number of oocytes retrieved (p-value-0.002) and pregnancy outcomes.

Broer $^{14}$ et al. performed a meta-analysis of 13 trials on AMH and 17 trials on AFC. They detected that sensitivities and specificities of $\mathrm{AMH}$ for the prediction of poor ovarian response varied between $40 \%$ and $91 \%$ and between $64 \%$ and $100 \%$, respectively. Moreover, the receiver operating characteristic (ROC) curves do not suggest a clearly better predictive ability for AMH than for AFC, and the difference was not statistically significant $(P=0.73)$. The authors concluded that $\mathrm{AMH}$ has at least the same level of accuracy and clinical value for the prediction of poor response and nonpregnancy as AFC. In our study we concluded that both serum AMH, AFC are good predictors of IVF/ICSI outcomes, AFC being better predictor than $\mathrm{AMH}$, serum $\mathrm{AMH}$ is statistically significant(p-value is 0.003 ) is less than 0.05 between pregnant and non pregnant women and AFC is highly significant statistically (p-value 0.0001) is less than 0.05 between pregnant and non pregnant women.

Prediction of poor ovarian reserve is not same as predicting ongoing pregnancy. In the study conducted by Sahmay $S^{15}$, et al, found that an AMH cut-off level of $2 \mathrm{ng} / \mathrm{ml}$ could predict poor response with a sensitivity of $78.9 \%$ and specificity of $73.8 \%$. In another study by Ocal $\mathrm{P}^{16}$, et al, found that an $\mathrm{AMH}$ cut-off level of $3.3 \mathrm{ng} / \mathrm{mL}$ predicted ovarian hyperstimulation syndrome (OHSS) with a sensitivity of $90 \%$ and a specificity of $71 \%$. However, depending on the 
results of the present study, we may speculate that it is not possible to determine a certain threshold of $\mathrm{AMH}$ that can predict ongoing pregnancy.

Mean serum AMH levels in pregnant groups $(5.1024 \mathrm{ng} / \mathrm{ml})$ is double that of non pregnant groups $(2.2738 \mathrm{ng} / \mathrm{ml})$ and mean AFC in pregnant (7.31) is double that of non pregnant (3.48) this suggests that both are good predictors of pregnancy outcomes and p-value of serum AMH is more than AFC this suggests that in our study AFC is better predictor of pregnancy than serum $\mathrm{AMH}$. In the present study, we also evaluated the clinical pregnancy rates according to the fertility potential. We observed that clinical pregnancy rates were more in low and satisfactory fertility potential. The pregnancy rate was $6.9 \%$ in the patients whose serum AMH level was lower than $2.1 \mathrm{ng} / \mathrm{ml}$ and $34.5 \%$ in patients whose serum AMH levels higher than $4 \mathrm{ng} / \mathrm{ml}$. The lowest level of serum AMH was $0.3 \mathrm{ng} / \mathrm{ml}$ in the pregnant group. Considering our results, we may suggest that $\mathrm{AMH}<1 \mathrm{ng} / \mathrm{ml}$ do not definitely predict conception failure.

To conclude, serum AMH levels and AFC are good predictors of pregnancy outcomes in IVF/ICSI cycles. The present study indicates that AFC is a better predictor of ovarian response compared to AMH. As AMH is an expensive test available at few places and AFC counts are measured by the infertility consultants, it can be used instead of $\mathrm{AMH}$ for predicting ovarian reserve and pregnancy outcome.

\section{Conflicts of interest}

The authors declare that there are no conflicts of interest

\section{Acknowledgements}

There are no acknowledgements

\section{BIBLIOGRAPHY}

1. Majumder K, Gelbaya TA, Laing I, Nardo LG. The use of anti-Müllerian hormone and antral follicle count to predict the potential of oocytes and embryos. Eur $\mathbf{J}$ Obstet Gynecol Reprod Biol. 2010;150 (2):166-70.

2. Nelson SM, Yates RW, Lyall H, Jamieson M, Traynor I, Gaudoin M, et al. Antimullerian Hormone based approach to controlled ovarian stimulation for assisted conception. Hum Reprod. 2009;24:867-75.

3. Gnoth C, Schuring AN, Friol K, Tigges J, Mallmann P, Godehardt E. Relevance of anti-Mullerian hormone measurement in a routine IVF program. Hum Reprod. 2008; 23: 1359-65.

4. Broer SL, Mol BW, Hendriks D, Broekmans FJ. The role of Antimullerian hormone in prediction of outcome after IVF : Comparison with the antral follicle count. Fertil Steril. 2009; 91: 705-14.

5. Aflatoonian A, Oskouian $\mathrm{H}$, Ahmadi S, Oskouian L. Prediction of high ovarian response to controlled ovarian hyperstimulation : Antimullerian hormone versus small antral follicle count $(2-6 \mathrm{~mm})$ J Assist Reprod Genet. 2009; 26: 319-25.

6. Freour T, Mirallie S, Colombel A, BachNgohou K, Masson D, Barriere P. Antimullerian hormone: Clinical relevance in assisted reproductive therapy. Ann Endocrinol. 2006; 67: 567-74.

7. Smeenk JM, Sweep FC, Zielhuis GA, Kremer JA, Thomas CM, Braat DD. Antimullerian hormone predicts ovarian responsiveness, but not embryo quality or pregnancy, after in vitro fertilization or intracytoplasmic sperm injection. Fertil Steril 2007; 87: 223-226. 
8. La Marca A, Stabile G, Artenisio AC, Volpe A. Serum anti-Mullerian hormone throughout the human menstrual cycle. Hum Reprod 2006; 21 (12): 3103-3107.

9. Visser J. Role of anti-Mullerian hormone in follicle recruitment and maturation. $\mathrm{J}$ Gynecol Obstet Biol Reprod (Paris) 2006; 35(5Pt2): 2S30-2S34.

10. Feyereisen E, Mendez Lozano DH, Taieb J, Hesters L, Frydman R, Fanchin R. AntiMullerian hormone: clinical insights into a promising biomarker of ovarian follicular status. Reprod Biomed Online 2006; 12(6): 695-703.

11. Hazout A, Bouchard P, Seifer DB, Aussage P, Junca AM, Cohen-Bacrie P. (2004) Serum antimüllerian hormone/ müllerianinhibiting substance appears to be a more discriminatory marker of assisted reproductive technology outcome than follicle-stimulating hormone, inhibin B, or estradiol. Fertil Steril.;82(5):1323-9.

12. Eldar-Geva T, Ben-Chetrit A, Spitz IM, Rabinowitz R, Markowitz E, Mimoni T, Gal M, Zylber-Haran E, Margalioth EJ. (2005) Dynamic assays of inhibin B, antiMullerian hormone and estradiol following FSH stimulation and ovarian ultrasonography as predictors of IVF outcome. Hum Reprod.;20(11):3178-83.

13. Wu CH, Chen $\mathrm{YC}, \mathrm{Wu} \mathrm{HH}$, Yang JG, Chang YJ, Tsai HD. (2009) Serum antiMüllerian hormone predicts ovarian response and cycle outcome in IVF patients. J Assist Reprod Genet.; 26(7): 383-9.
14. Broer SL, Mol BW, Hendriks D, Broekmans FJ. (2009) The role of antimullerian hormone in prediction of outcome after IVF: comparison with the antral follicle count. Fertil Steril.;91(3): 705-14.

15. Sahmay S, Cetin M, Ocal P, Kaleli S, Senol H, Birol F, Irez T. Serum antiMüllerian hormone level as a predictor of poor ovarian response in IVF patients. Reprod Med Biol. 2011; 10:9-14.

16. Ocal P, Sahmay S, Cetin M, Irez T, Guralp O, Cepni I. Serum anti-Müllerian hormone and antral follicle count as predictive markers of OHSS in ART cycles. J Assist Reprod Genet. 2011;28 (12):1197-203.

17. Ibrahim AA, Maha MB, Hanan HM. AntiMullerian and antral follicle count as predictors of ovarian reserve and successful IVF. Asian Pacific J of Reprod 2012; 1(2): 89-92.

18. Wang JG, Douglas NC, Nakhuda GS, Choi JM, Park SJ, Thornton MH, Guarnaccia MM, Sauer MV. The association between anti- Müllerian hormone and IVF pregnancy outcomes is influenced by age. Reprod Biomed Online. 2010 Dec;21(6):757-61.

19. Kini S, Li HW, Morrell D, Pickering S, Thong KJ. Anti-mullerian hormone and cumulative pregnancy outcome in in-vitro fertilization. J Assist Reprod Genet. 2010; 27: 449-56. 